\title{
An Investigation of Image Segmentation Method for Remotely Sensed Hyperspectral Images with Region Object Aggregations
}

\author{
A. Nirmala \\ Ph.D Scholar, \\ Assistant Professor(SG) \\ Dr.N.G.P Arts \& Science College
}

\begin{abstract}
An important aspect of spectral image analysis is identification of materials present in the object or scene being imaged. Since multi-spectral or hyper spectral imagery is generally low resolution, it is possible for pixels in the image to contain several materials. A paramount issue in image processing area is to design and implement an efficient segmentation and classification techniques demanding optimal resources. This paper presents a survey on all prominent region growing segmentation techniques analyzing each one and thus sorting out an optimal and promising technique. Finally study the importance of the best merge region growing normally produces segmentations with closed connected region objects. Recognizing that spectrally similar objects often appear in spatially separate locations, present an approach for tightly integrating best merge region growing with nonadjacent region object aggregation, which we call hierarchical segmentation or HSeg. The effectiveness of the proposed methodology is illustrated by comparing its performance with the state-of-the-art methods on synthetic and real hyper spectral image data sets. The reported results give clear evidence of the relevance of using both spatial and spectral information in hyper spectral image segmentation.
\end{abstract}

\section{Keywords}

Image analysis, hyper spectral images, image classification, image region analysis, image segmentation, objects detection.

\section{INTRODUCTION}

Hyper spectral image segmentations can reveal spatial trends that show the physical structure of the scene to an analyst. They highlight borders and reveal areas of homogeneity and change. The major objective of the hyper spectral image Segmentations are independently helpful for object recognition, and assist with automated production of symbolic maps. Additionally, a good segmentation can dramatically reduce the number of effective spectra in an image, enabling analyses that would otherwise be computationally prohibitive. Segmentation of high dimensional data sets such as remotely sensed hyperspectral images is a difficult endeavor [1]. This brings about two problems. First, the access to a consistent set with a sufficient number of training samples is often impossible or highly costly. Second, the use of large training sets in high dimensional spaces leads to expensive computational demands. In particular, using an over segmentation of the image instead of individual pixels can reduce noise and potentially improve the results of statistical post-analysis.

While pixel wise classification techniques process each pixel independently without considering information about spatial structures [2], further improvement of classification results can be achieved by considering spatial dependencies between pixels, i.e., by performing spectral-spatial classification [3-4]. While this analysis approach can be satisfactory for some applications, it is usually not fully effective in extracting the information content from remotely sensed imagery, especially from high spatial resolution imagery .

Recent work in hyper spectral image segmentation includes the watershed transform [5], Markov Random Fields [6]. Generally speaking, these techniques cluster pixels based on spatial proximity and a measure of spectral similarity. Existing hyper spectral segmentation approaches generally use task-agnostic distance measures that treat all channels equally or weight them based on global statistical properties of the dataset. Such metrics are often confused by noise, instrument artifacts, or spectral variations that are irrelevant to semantic categories of interest.

Learning a task-specific similarity metric from labeled data can ameliorate this problem. Methods to learn such metrics include Information Theoretic Metric Learning (ITML) [7] and variants of Generalized Relevance Learning Vector Quantization (GRLVQ) [8]. But these methods still have some of the problems as mentioned above in order to solve this problem in this work focus on the field of object-based image analysis (OBIA) has arisen in recent years to address the need to move beyond pixel-by-pixel analysis [9]. Image segmentation is the first step for most OBIA approaches and is a key factor in determining the level of performance for these image analysis approaches.

A popular approach for performing image segmentation is best merge region growing. Beaulieu and Goldberg's hierarchical stepwise optimization (HSWO) is an iterative form of region growing, in which the iterations consist of finding the most optimal or best segmentation with one region less than the current segmentation. In this work study of the existing image segmentation methods for hyper spectral image data and major finding of these image segmentation methods and how to solve these problems also specified in the conclusion and future works.

\section{BACKGROUND STUDY}

Watershed transformation is a powerful morphological approach to image segmentation which combines region growing and edge detection. The watershed is usually applied to the gradient function, and it divides an image into regions, so that each region is associated with one minimum of the gradient image. The extension of a watershed technique to the case of hyper spectral images has been investigated in [10]. One of the drawbacks of the Color Morphological Gradient (CMG) is that it is very sensitive to noise. As a result, the image is partitioned into a set of regions, and one subset of watershed pixels, i .e., pixels situated on the borders between regions. Finally, every watershed pixel is assigned to 
the neighboring region with the "closest" median $\mathrm{z}$ (the distance between the vector median of this region and the watershed pixel is minimal).

The Expectation Maximization (EM) algorithm for the Gaussian mixture resolving belongs to the group of partitional clustering techniques [4]. The use of partitional clustering for hyperspectral image segmentation has been discussed in [4]. Clustering aims at finding groups of spectrally similar pixels. When the algorithm converges, the partitioning of the set of image pixels into clusters is obtained. However, as no spatial information is used during the clustering procedure, pixels with the same cluster label can form a connected spatial region, or can belong to disjoint regions. In order to obtain segmentation map, a connected components labeling algorithm is applied to the output image partitioning obtained by clustering.

Jun Li et al [11] proposed a Supervised segmentation algorithm for remotely sensed hyperspectral image data which integrates the spectral and spatial information in a Bayesian framework. A multinomial logistic regression (MLR) algorithm is first used to learn the posterior probability distributions from the spectral information, using a subspace projection method to better characterize noise and highly mixed pixels. Then, contextual information is included using a multilevel logistic Markov-Gibbs Markov random field prior. Finally, a maximum a posteriori segmentation is efficiently computed by the $\alpha$-Expansion mincut- based integer optimization algorithm. The proposed segmentation approach is experimentally evaluated using both simulated and real hyper spectral data sets, exhibiting state-of-the-art performance when compared with recently introduced hyper spectral image classification methods. The integration of subspace projection methods with the MLR algorithm, combined with the use of spatial-contextual information, represents an innovative contribution in the literature. The major findings of the works are that high dimensional data problem are not solved during segmentation and object detection based segmentation not performed.

Tarabalka et al. [12] present an SVM- and MRF-based method that comprises two steps: First, a probabilistic SVM pixelwise classification of the hyper spectral image is performed, followed by MRF-based regularization for incorporating spatial and edge information into the classification. Another example of a Markov-based classification framework is presented in [13] where a neuro fuzzy classifier is used to perform classification in the spectral domain and compute a first approximation of the posterior probabilities, and the resulting output is then fed to an MRF spatial analysis stage combined with a maximum likelihood (ML) probabilistic reclassification.

In addition to MRF-based approaches, extended morphological profiles were also considered to integrate spatial information in the classification of hyperspectral images [13], as well as a composite kernel methodology [14]. Another approach considered consists in performing segmentation and pixelwise classification independently and then combining the results using a majority voting rule where a watershed technique has been used to perform segmentation and an SVM pixelwise classification is performed, followed by majority voting in the watershed regions. The major issue of the work is that perform image segmentation for region based object only ,no region based object detection is not performed.
The construction of minimum spanning tree algorithm extends to hyperspectral images [4]. This is similar to the marker controlled segmentation approach, this algorithm works out to generate classification derived markers and do segmentation and classification processing based on the markers. An improvement is that instead of using a gradient, the image and constructing a minimum spanning forest (MSF) where markers are the roots. A polling technique is implemented to identify connected components which work together with the minimum spanning forest to generate optimal segmentation and classification map. A demerit of this method is that if no marker is chosen for a particular spatial structure, this spatial region will be lost in the final classification map. A merit of this method is that improves classification accuracies. A demerit of this method is that if no marker is chosen for a particular spatial structure, this spatial region will be lost in the final classification map.

The Hierarchical image SEGmentation (HSEG) algorithm is a segmentation technique based on iterative hierarchical stepwise optimization region growing method. Furthermore, it provides a possibility of merging non-adjacent regions by spectral clustering [15]. The following outline of the HSEG algorithm is based on the description given in [15] .In order to reduce computational demands, a Recursive divide-andconquer approximation of HSEG (RHSEG) has been developed. The NASA-Goddard RHSEG software provides an efficient implementation of the RHSEG algorithm. When determining most similar pair of regions, propose to choose the standard Spectral Angle Mapper (SAM) between the region mean vectors and as the dissimilarity criterion [15].

This hierarchical sequence allows flexibility in choosing the appropriate level of detail for the segmentation map. When training data is available, it is a simple process to quantitatively evaluate the segmentation results at each hierarchical level versus the training data to select the appropriate level of detail. Otherwise an appropriate level of segmentation detail can be chosen interactively with the program HSEG Viewer. Unfortunately, the approach taken for spatially disjoint region object aggregation requires excessive computing time in the original formulation of HSeg.

Fang Lia et al[16] first describe a variational fuzzy segmentation model coupled with a denoising/deblurring model for material identification. A particularly simple segmentation approach makes use of the spatial variation of spectral correlation in a typical hyperspectral dataset. Specifically, since according to the physical model all spatial pixels of a given material in an image have the same normalized spectral trace, a coarse-grained spectral correlation function computed over a small moving 2D spatial cell of fixed shape and size will show sharp variations as the averaging cell crosses a boundary between two materials. A statistical moving average method for segmentation is also described, object detection is not carried out in this work.

\section{FINDINGS OF THE SURVEY AND SOLUTIONS}

The major findings of the existing image segmentation in the literature are detaliy specified in following section,

- The extension of a watershed technique to the case of hyperspectral images has been investigated in [10]. One of the drawbacks of the Color Morphological Gradient $(\mathrm{CMG})$ is that it is very sensitive to noise.

- The Expectation Maximization (EM) algorithm for the Gaussian mixture resolving belongs to the group of 
partitional clustering techniques [4]. However, as no spatial information is used during the clustering procedure, pixels with the same cluster label can form a connected spatial region, or can belong to disjoint regions.

- Jun Li et al [11] proposed a Supervised segmentation algorithm for remotely sensed hyperspectral image data which integrates the spectral and spatial information in a Bayesian framework.

- The major findings of the works are that high dimensional data problem are not solved during segmentation and object detection based segmentation not performed.

- MRF-based approaches, extended morphological profiles were also considered to integrate spatial information in the classification of hyperspectral images [13]. The major issue of the work is that perform image segmentation for region based object only ,no region based object detection is not performed.

- The construction of minimum spanning tree algorithm extends to hyperspectral images [4]. A demerit of this method is that if no marker is chosen for a particular spatial structure, this spatial region will be lost in the final classification map.

- The Hierarchical image SEGmentation (HSEG) algorithm is a segmentation technique based on iterative hierarchical step-wise optimization region growing method[15]. Unfortunately, the approach taken for spatially disjoint region object aggregation requires excessive computing time in the original formulation of HSeg.

- A statistical moving average method [16] for segmentation is also described, object detection is not carried out in this work.

\subsection{Solutions}

A form of best merge region-growing segmentation lies at the core of the segmentation approach contained in eCognition 2.1 [14], [15]. However, the process for selecting the best merges is much more involved than the relatively straightforward evaluation and comparison of region .But in this work still selection of the region and non region portions after the image segmentation becomes still difficult and noise regions are not removed during segmentation .Our future work focus on to solve these problems by proposing the classification methods for region selection and filtering methods for noise removal .Then improved region growing segmentation is performed in future.

Please use a 9-point Times Roman font, or other Roman font with serifs, as close as possible in appearance to Times Roman in which these guidelines have been set. The goal is to have a 9-point text, as you see here. Please use sans-serif or non-proportional fonts only for special purposes, such as distinguishing source code text. If Times Roman is not available, try the font named Computer Modern Roman. On a Macintosh, use the font named Times. Right margins should be justified, not ragged.

\section{EXPERIMENTATION RESULTS}

Timing tests comparing the original and refined versions of HSeg were performed on portions of a tow real time datasets .These tests also include timing tests for RHSeg incorporating either the original or refined version of HSeg. The square root of the band sum mean squared error (BSMSE) dissimilarity criterion was utilized with $8 \mathrm{nn}$ neighborhoods and no small region merge acceleration. For very small images (such as 64 $\times 64$ pixels), the wall clock run times for the two versions are similar. As the image sizes get larger, the run times of the two versions diverge more and more until, at an image size of 256 $\times 256$ pixels, the refined version of HSeg is from 12 to over 93 times faster than the original version.

In order to demonstrate the effectiveness of the new versions of HSeg and RHSeg, we now turn to the problem of evaluating image segmentation quality. Then have chosen to assess segmentation results using a region-based classification approach [4]. The first step is to perform a pixelwise classification of an image data set. Then, a region classification is obtained by assigning each spatially connected region from the segmentation result to the most frequently occurring class within the region.

\section{University of Pavia ROSIS Data Set}

The color samples which is taken for hyperspectral image segmentation are also shown in Fig.1 and Fig.2. The University of Pavia data set was recorded by the Reflective Optics System Imaging Spectrometer (ROSIS) over the

University of Pavia, Pavia, Italy. The image is $610 \times 340$ pixels in size, with a spatial resolution of $1.3 \mathrm{~m}$. The ROSIS sensor has115 spectral channels, with a spectral range of $0.43-0.86 \mu \mathrm{m}$. The 12 noisiest channels were removed, and the remaining 103 spectral bands were used in this experiment. The reference data contain nine

ground cover classes: asphalt, meadows, gravel, trees, metal sheets, bare soil, bitumen, bricks, and shadows. A three-band false color image of this data set and the ground reference data are shown in Fig 1.

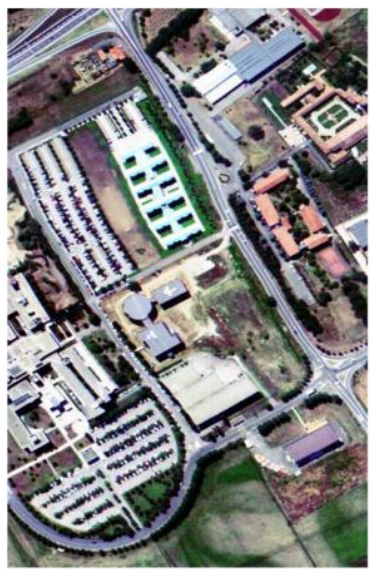

(a)

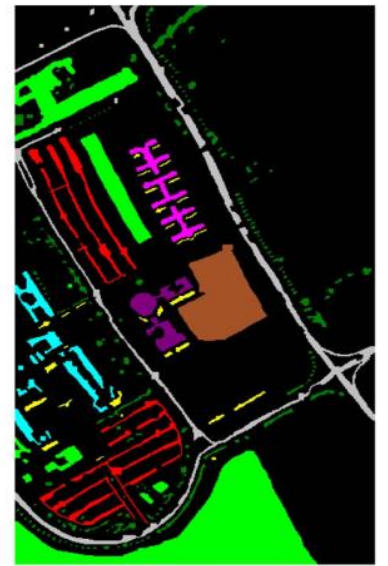

(b)

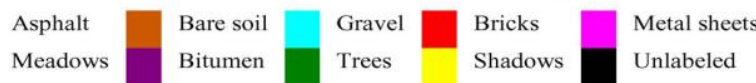

(c)

Fig. 1. (a) Three-band false color image of the University of Pavia hyper spectral data set (RGB = bands 56, 33, and 13). (b) Reference data. (c) Color key. 


\subsection{Indian Pines AVIRIS Hyper spectral Data Set}

The Indian Pines hyperspectral data set was obtained through Purdue University's MultiSpec freeware project [19]. This data set was acquired by the AVIRIS sensor over the vegetated Indian Pines site in Northwestern Indiana. The image has spatial dimensions of 145 by 145 pixels, with a ground spatial resolution of $20 \mathrm{~m} /$ pixel. Twenty water absorption bands (104-108,150-163, and 220) were removed , resulting in a 200-band image that was used in our experiments. The ground reference data contain 16 classes of interest, which represent mostly different types of crops. A three-band false color image and the reference data are shown in Fig. 2.

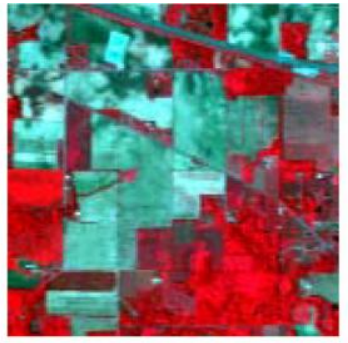

(a)

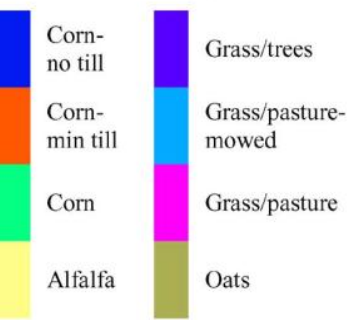

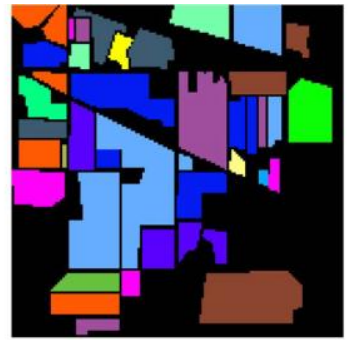

(b)

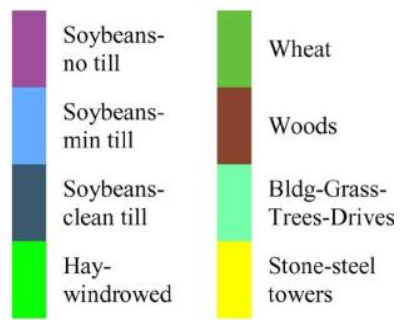

(c)
Fig. 2. (a) Three-band false color image of the Indian Pines hyperspectral data set (RGB = bands 47, 24, and 14). (b) Reference data. (c) Color key (black designates unlabeled)

Table 1: Comparison Of Classification Accuracies For proposed hybrid refined HRHSeg ,HSeg and RHSeg

\begin{tabular}{|c|c|c|c|}
\hline Methods & $\begin{array}{c}\text { Proposed } \\
\text { hybrid } \\
\text { refined HSeg }\end{array}$ & RHseg & Hseg \\
\hline No of regions & 56882 & 220122 & 20991 \\
\hline $\begin{array}{c}\text { Overall } \\
\text { accuracy(\%) }\end{array}$ & 97.5 & 96.7 & 95.2 \\
\hline $\begin{array}{c}\text { Average } \\
\text { accuracy (\%) }\end{array}$ & 97.2 & 96.2 & 95.1 \\
\hline $\begin{array}{c}\text { Kappa } \\
\text { coefficient (\%) }\end{array}$ & 96.45 & 95.2 & 94.3 \\
\hline Roofs & 93.2 & 91.2 & 90.3 \\
\hline Street & 94.9 & 92.4 & 91.8 \\
\hline Graveled & 95.6 & 94.2 & 93.8 \\
\hline Path & 98.24 & 96.5 & 95.8 \\
\hline Grass & 98.56 & 97.45 & 96.4 \\
\hline Trees & 96.7 & 95.8 & 95.2 \\
\hline Water & 99.2 & 97.7 & 97.2 \\
\hline Shadow & 96.45 & 94.5 & 94.1 \\
\hline
\end{tabular}

\section{CONCLUSION AND FUTURE WORK}

Hyper spectral sensors capture images in hundreds of narrow spectral channels. The detailed spectral signatures for each spatial location provide rich information about an image scene, leading to better discrimination between physical materials and objects. However, interpretation of these highdimensional signatures and identification of objects is a challenging task. Although pixelwise classification techniques have been proposed to solve image segmentation problems when dealing with hyperspectral data, the incorporation of the spatial context into classification procedures yields further improvement of the acc uracies. But these methods doesn't perform the non adjacent based object detection for segmentation of hyperspectral images samples and this problem is solved by using merge region-growing segmentation that tightly integrates nonadjacent region object aggregation with the usual region object growing. This work was outset from the need to study and analyze various region growing image segmentation techniques on hand. A keen analysis is done on each approach to juice out their features and overheads. A major finding is that most of the techniques impose a profound level of complexity. Thus, a prominent work can be one which integrates parallel processing paradigm into the available image processing techniques to enhance the overall accuracy of the work practice. Kittle, et al. [20] indicates that more than one frame of measurements may improve the robustness of reconstruction/ segmentation against noise, and warrants further investigation.

\section{ACKNOWLEDGMENTS}

A.Nirmala working as an Assistant Professor(SG) in Dr.N.G.P Arts and Science College, Coimbatore.

\section{REFERENCES}

[1] D. Landgrebe, Signal Theory Methods in Multispectral Remote Sensing. Hoboken, NJ: Wiley, 2003

[2] J.-M. Yang, P.-T. Yu, and B,-C. Kuo, "A nonparametic feature extraction and its application to nearest neighbor classification for hyperspcetral image data:' IEEE Trans. Geos. and Remote Sens., vol. 48, no. 3, pp, 1279-1293, March 2010.

[3] M. Fauvel, J. Chanussot. J. A. Benediktsson, and J. R. Sveinsson, "Spectral and spatial classification of hyperspectral data using SVMs and morphological profilers." IEEE Trans. Geos. and Remote Sensvol. 46, no. 10 , Oct. 2008.

[4] Y, Tarabalka, J. A. Benediktsson, and J. Chanussot, "Classification of hyperspectral data using Support Vector Machines and adaptive neighborhoods," in Prot. of the 61h E,ARSeL SIC, IS workshop, Tel Aviv, Israel, 2009.

[5] Y. Tarabalka, J. Chanussot, and J.A. Benediktsson, "Segmentation and classification of hyperspectral data using watershed transformation," Pattern Recognition, vol. 43, no. 7, pp. 2367-2379, 2010

[6] Mohammadpour, O. F'eron, and A. Mohammad-Djafari, "Bayesian segmentation of hyperspectral images," Bayesian Inference and Maximum Entropy Methods in Science and Engineering, vol. 735, pp. 541-548, 2004.

[7] J Davis, B Kulis, P Jain, S Sra, and I Dhillon, "Information theoretic metric learning," Proceedings of the 24th international conference on Machine learning, Jan 2007. 
[8] M.J Mendenhall and E Mer'enyi, "Relevance-based feature extraction for hyperspectral images," Neural Networks, IEEE Transactions on, vol. 19, no. 4, pp. 658672,2008

[9] T. Blaschke, S. Lang, and G. J. Hay, Eds., Object-Based Image Analysis: Spatial Concepts for Knowledge-Driven Remote Sensing Applications. Berlin, Germany: Springer-Verlag, 2008.

[10] Tarabalka, J. Chanussot, J. A. Benediktsson, J. Angulo, and M. Fauvel, "Segmentation and classification of hyperspectral data using watershed," in Proc. of IGARSS 'CS, Boston, USA, 2008 ; pp. 111 -652 111-655.

[11] Jun Li, José M. Bioucas-Dias and Antonio Plaza, "Spectral-Spatial Hyperspectral Image Segmentation Using Subspace Multinomial Logistic Regression and Markov Random Fields" IEEE TRANSACTIONS ON GEOSCIENCE AND REMOTE SENSING, VOL. 50, NO. 3, MARCH 2012,pp.809-823.

[12] Y. Tarabalka, M. Fauvel, J. Chanussot, and J. Benediktsson, "SVM and MRF-based method for accurate classification of hyperspectral images," IEEE Geosci. Remote Sens. Lett., vol. 7, no. 4, pp. 736-740, Oct. 2010.

[13] A. Plaza, J. A. Benediktsson, J. W. Boardman, J. Brazile, L. Bruzzone, G. Camps-Valls, J. Chanussot, M. Fauvel, P. Gamba, A. Gualtieri, M. Marconcini, J. C. Tilton, and G. Trianni, "Recent advances in techniques for hyperspectral image processing," Remote Sens. Environ., vol. 113, pp. 110-122, Sep. 2009.

[14] G. Camps-Valls, L. Gomez-Chova, J. Munoz-Mari, J. Vila-Frances, and J. Calpe-Maravilla, "Composite kernels for hyperspectral image classification," IEEE Geosci. Remote Sens. Lett., vol. 3, no. 1, pp. 93-97, Jan. 2006.

[15] J. C. Tilton, "HSEG/RHSEG, HSECr Viewer and RSEG Reader user's manual (version 1,40)," Provided with the evaluation version c!f RHSEG available from: http: Ilipp.gsfc.nasa.grn/RHSEG, 2008.

[16] F. Li, M. Ng, R. Plemmons, S. Prasad, and Q. Zhang, "Hyperspectral image segmentation, deblurring, and spectral analysis for material identification," in Proc. SPIE Conf. on Defense, Security and Sensing, Vol. 7701, 2010

[17] M. Baatz and A. Schape, "Multiresolution segmentation: An optimizing approach for high quality multi-scale segmentation," in Angewandte Geographich Informationsverarbeitung, XII, J. Strobl and T. Blaschke, Eds. Heidelberg, Germany: Wichmann, 2000, pp. 12-23.

[18] U. Benz, P. Hofmann, G. Wilhauck, I. Lingenfelder, and M. Heynen, "Multi-resolution, object-oriented fuzzy analysis of remote sensing data for GIS-ready information," ISPRS J. Photogrammm. Remote Sens., vol. 58, no. 3/4, pp. 239-258, Jan. 2004.

[19] D. A. Landgrebe, Signal Theory Methods in Multispectral Remote Sensing. Hoboken, NJ: Wiley, 2003.

[20] D. Kittle, K. Choi, A. Wagadarikar, and D. Brady, "Multiframe image estimation for coded aperture snapshot spectral imagers," Applied Optics, vol. 49, pp. 6824-6833, 2010. 